\title{
To Use or not to Use Anticoagulation in Patients with Advanced Malignancies? - This is the Question
}

\author{
Running title: Should we use a more flexible approach to cancer-associated thrombosis?
}

\section{Katarzyna Rygiel ${ }^{\star}$}

Department of Family Practice, Medical University of Silesia (SUM), Zabrze, Poland.

*Corresponding author: Katarzyna Rygiel, Email: kasiaalpha@yahoo.co.uk

\section{Abstract}

The management of cancer-associated thrombosis (CAT) often presents challenges to members of medical teams, attending to patients with advanced malignancies. In general, indications for treatment of CAT have been driven by the randomized clinical trials (RCTs) that have usually excluded patient populations suffering from metastatic cancers, at the end-of-life period. Since the findings of such RCTs are not representative of this particular group of oncology patients, medical providers need practical help in the daily care of this population.

It should be noted that venous thromboembolism (VTE) associated with cancer has been related to increased morbidity and mortality rates, as well as different symptoms that can deteriorate the patient's quality of life (QoL). Therefore, current guidelines promote the use of anticoagulation therapy in oncology patients, focusing on the acute care contexts (e.g., a perioperative period, or chemotherapy (CHT) regimen).

In contrast, in the palliative care (PC) setting, the main benefit of anticoagulation should be a reduction of symptom burden associated with VTE. However, during end-of-life care, the risk of bleeding can often overweight the potential benefits of anticoagulation.

To shed some light on these complicated issues, this mini-review presents some challenges in CAT anticoagulation management. It briefly discusses VTE risk factors and therapeutic options and compares acute and PC services. This overview supports therapeutic teams in charge of oncology patients receiving PC services, where the main goal is not life extension but an assurance of the best possible QoL.

\section{Keywords}

Cancer-associated thrombosis (CAT); Low molecular weight heparin (LMWH); Novel oral anticoagulants (NOACs); Palliative care (PC); Quality of life (QoL); Venous thromboembolism (VTE) 


\section{INTRODUCTION}

Due to remarkable progress in medical sciences, many patients with advanced malignancies achieve now a longer survival than even before. However, the management of such patients, especially at the border of active and palliative care $(\mathrm{PC})$, requires personalized attention, precise medical guidelines, and reasonable decision-making, integrating many aspects of oncology care.

Venous thromboembolism (VTE) that includes a deep venous thrombosis (DVT) and a pulmonary embolism (PE) represents a potentially preventable mortality reason for many hospital patients ${ }^{[1]}$. Notably, patients with cancer are at particular risk of VTE, and thus, clinical guidelines recommend pharmacological thromboprophylaxis for such patients, who are hospitalized due to acute illness ${ }^{[2]}$. However, these recommendations are extrapolated from thromboprophylaxis trials that were not conducted specifically among patients with cancer ${ }^{[3]}$. Moreover, such trials usually did not take into consideration dynamic changes of thrombogenesis, especially during the malignancy progression ${ }^{[3]}$. Notably, patients with advanced cancers and a life expectancy of less than three months were also excluded from these studies ${ }^{[3]}$. Nevertheless, in reality, many patients with advanced, incurable cancers, upon their admission to hospital, were still given a routine thromboprophylaxis ${ }^{[4]}$. In contrast, only a small number of such patients were admitted to specialist PC units, in which providing comfort has been the primary goal of care (e.g., via symptom control, and not by prolonging survival), while thromboprophylaxis has not been the main priority ${ }^{[5]}$. In fact, VTE in the PC setting is considered to be clinically relevant, only if it is in agreement with a patient-reported symptom burden or if it contributes to discomfort at the end of life ${ }^{[6]}$. Among patients with malignancies, the risk of VTE has been augmented by the progression of cancer to advanced or metastatic stages, poor performance status, past history of thrombotic complications, and a plethora of different factors, relevant to both the tumor and the patient clinical characteristics ${ }^{[7]}$. Since VTE has been associated with increased morbidity and mortality rates, current guidelines promote the use of anticoagulation therapy or prophylaxis in oncology patients, focusing on the acute care contexts (e.g., a perioperative period, or chemotherapy (CHT) regimen) ${ }^{[7]}$. VTE related to cancer has been associated with various symptoms that can deteriorate the patient's quality of life (QoL) (e.g., shortness of breath, chest pain, hemoptysis, leg pain) ${ }^{[7]}$.

However, during the end-of-life care, advantages of anticoagulation are uncertain, and risks of bleeding may overweight its potential benefits. This is particularly important, when the bleeding adverse effects can be additionally magnified by conditions, which are commonly seen at the end-of-life period, such as dehydration, cachexia, renal or hepatic failure ${ }^{[8]}$. On the one hand, in the end-of-life care situation, when providing the most optimal QoL is the top priority, VTE prophylaxis may be desirable to reduce the symptom burden ${ }^{[8]}$. On the one hand, however, randomized clinical trials (RCTs) addressing the proportions between the benefits and the risks of anticoagulation in patients approaching the final stage of life are very limited ${ }^{[8]}$.

Currently, some observational studies have provided valuable input into the dilemma of CAT management, among oncology patients in the PC setting. For instance, according to a recent study, it appears that DVT is related to only a limited symptom burden, and has no definite impact on shortening of the final period of life, in the population with advanced malignancies ${ }^{[9]}$.

This mini-review addresses some challenges in CAT anticoagulation management. It discusses VTE risk factors and common therapeutic options. Moreover, this paper compares acute and PC settings, and focuses on the QoL, at the end-of-life period. This overview provides guidance and support in the area of VTE prophylaxis to therapeutic teams in charge of oncology patients with advanced malignancies, especially at the intersection of the active and supportive type of care, when the QoL becomes a priority over the extending life.

\section{LESSONS LEARNED FROM THE HIDDEN STUDY}

The prevalence of DVT in patients with advanced malignancies has been unknown, and it remains unclear whether 
or not the standard recommendations for thromboprophylaxis are applicable to such patients. To elucidate this issue, commonly encountered in patient population of the PC services the HIDDen study was conducted to determine the prevalence and predictors of femoral DVT, in patients admitted to PC services (e.g., hospices or specialist PC units) ${ }^{[9]}$. The HIDDen was a prospective, longitudinal, observational study that has explored the prevalence, symptom burden, and natural history of DVT in patients with advanced cancers at the PC setting. It has been noted that VTE in the PC setting is considered as clinically relevant, only if it augments a symptom burden or deteriorates the patient's OoL at the terminal stage ${ }^{[9]}$. Consequently, the HIDDen study results suggest that the DVT does not aggravate a patient's discomfort and does not shorten lifespan. Moreover, this study begs a question about the specific place of pharmacological thromboprophylaxis in such patients. In addition, the study highlights certain points that can challenge some current recommendations for prevention of VTE in patients with advanced malignancies. In short, the HIDDen study involved 343 patients with advanced cancers (those with an estimated prognosis below five days were excluded), who were admitted to PC services in the United Kingdom, and evaluated for proximal DVT (by using bilateral ultrasonography (USG) of the popliteal and femoral veins) ${ }^{[9]}$. Over 30\% of the study participants were found to have a femoral vein thrombosis, and a lower-extremity (LE) edema (that was an independent predictor of DVT). Notably, in the HIDDen study group, there was no association between the use of thromboprophylaxis and the risk for DVT on admission to PC services. Furthermore, having a DVT had no influence on mean survival time (e.g., for patients with DVT on admission, survival was approximately one month) ${ }^{[9]}$. Also, it has been reported that about $25 \%$ of patients, who did not have history of DVT on admission, were newly diagnosed with DVT, after being admitted to the PC services. It should be underscored that except from LE edema, the study results have not revealed any effect of femoral vein thrombosis on experience of VTE-related symptoms, nor effect of thromboprophylaxis on DVT risk ${ }^{[9]}$. The HIDDen study results have revealed that the prevalence of DVT in patients with advanced cancer was very high. However, since DVT in many of these patients did not cause symptoms, in a setting where symptom relief (rather than the extension of life) is the main priority, providing the patient's comfort is of utmost importance ${ }^{[9]}$. Moreover, according to the recent ASCO guidelines, the anticoagulation is of uncertain benefit among oncology patients with poor prognosis, whose life expectancy is short, and in cases, in which anticoagulants do not decrease the symptom burden ${ }^{[7]}$. Such findings suggest that VTE is a manifestation of advanced cancer and that thromboprophylaxis plays only a very limited role in this context. Therefore, it should be critical for clinicians to reduce the unnecessary risk of thromboprophylaxis (e.g., bleeding), unless there would be objective benefits, depending on the individual patient's scenario ${ }^{[7,8,9]}$. In addition, in the HIDDen study, a significant predictor for DVT was a bedrest within the last three months, suggesting that the thrombotic events could have occurred prior to PC admission (due to an impending decline of the patient's status, and not as the cause of it) ${ }^{[9]}$. Certainly, the HIDDen study offers a new perspective to the approach to thromboprophylaxis in patients with cancer, at the end of life care ${ }^{[9]}$.

\section{CONSIDERATIONS OF THROMBOPROPHYLAXIS IN PATIENTS WITH ADVANCED CANCERS}

Strategies to prevent hospital-acquired thrombosis are a medical priority, and thus, patients with advanced cancers represent a target population, since they are highly thrombotic and almost $90 \%$ of them have been estimated to have five hospital admissions in their final year of life ${ }^{[10]}$. It should be kept in mind that a decision about the application of thromboprophylaxis, to a large degree, depends on the type of admitting facility. For instance, thromboprophylaxis is more likely to be administered in an acute care hospital, compared to a PC setting. Therefore, revisiting the issue of pharmacological thromboprophylaxis, especially in the PC inpatient population, from a perspective of procedures that are mandatory at a given medical facility, would be useful.

Notably, the clinical decision to admit certain oncology patients to the PC setting should be based on several components, such as an advanced stage of the neoplastic disease and short prognosis, poor performance status, elderly age, as well as an individual patient's preferences, in addition to a physician's clinical judgment with regard to the patient's overall condition and expected outcome ${ }^{[8]}$. In particular, when choosing the admission facility, clinicians 
should keep in mind a differential diagnosis spectrum, including for DVT: lymphedema, cellulitis, or hypoalbuminemia, and for PE: pneumonia, pleural effusion, heart failure, lung metastases, or lymphangitis. In the acute care hospital, in which patients are evaluated for VTE risk, and then are given VTE prophylaxis (when indicated), it is critical to assess a possible reversibility or potential improvement of conditions that were the main reasons for hospitalization ${ }^{[7]}$. In general, patients with advanced cancers, admitted to an acute hospital setting represent a diversified population with regard to their prognosis and performance status. Due to that, knowing their demographic and medical characteristics is essential to make the most appropriate therapeutic efforts in a given clinical context (e.g., for patients with a good performance status, or with only a temporary increase of the pro-thrombotic risk factors).

Another valid question is whether or not thromboprophylaxis should be administered before developing the advanced neoplastic disease. For instance, according to the FRAGMATIC trial that has explored standard CHT plus LMWH prophylaxis, in patients with newly diagnosed lung cancer, no improvement of survival was achieved ${ }^{[11]}$. Similarly, in a study examining gemcitabine vs. gemcitabine plus LMWH (dalteparin) thromboprophylaxis, in patients with pancreatic cancer, survival was also not improved ${ }^{[12]}$. However, it should be noted that the application of a highdose LMWH had prevented a lethal PE among this study participants ${ }^{[12]}$.

\section{REFINEMENTS OF INDICATIONS FOR ANTICOAGULATION THERAPY IN PATIENTS WITH CANCER-RELATED VENOUS THROMBOEMBOLISM}

Table 1. Pharmacologic interactions between some commonly used antineoplastic therapies and novel oral anticoagulants

\begin{tabular}{|c|c|c|c|c|}
\hline \multicolumn{5}{|c|}{ Pharmacologic interactions between antineoplastic therapies and novel oral anticoagulants ${ }^{[15]}$} \\
\hline NOAC & \multicolumn{2}{|c|}{$\begin{array}{l}\text { Anticancer agents inducing } \\
\text { P-glycoprotein \& CYP3A4 } \\
\text { - causing a decrease of NOAC's levels }\end{array}$} & \multicolumn{2}{|c|}{$\begin{array}{l}\text { Anticancer agents inhibiting } \\
\text { P-glycoprotein \& CYP3A4 } \\
\text { - causing an increase of NOAC's levels }\end{array}$} \\
\hline Endoxaban & \begin{tabular}{|l|} 
CHT \\
• Doxorubicin \\
・ Vinblastine \\
HT (AA) - Enzalutamide \\
ST - Dexamethasone \\
\end{tabular} & \begin{tabular}{|l} 
Inducing \\
P-glycoprotein
\end{tabular} & \begin{tabular}{|l|} 
TT (TKI) \\
• Lapatinib \\
$\bullet$ Nilotinib \\
• Sunitinib \\
HT (ERA) - Tamoxifen \\
\end{tabular} & $\begin{array}{l}\text { Inhibiting } \\
\text { P-glycoprotein }\end{array}$ \\
\hline Apixaban & \begin{tabular}{|l|} 
CHT \\
• Doxorubicin \\
• Vinblastine \\
HT (AA) - Enzalutamide \\
ST - Dexamethasone
\end{tabular} & $\begin{array}{l}\text { Inducing } \\
\text { P-glycoprotein \& CYP3A4 }\end{array}$ & \begin{tabular}{|c|} 
TT (TKI) \\
$\cdot$ Imatinib \\
$\cdot$ Dasatinib \\
$\cdot$ Lapatinib \\
• Nilotinib \\
• Sunitinib \\
HT (ERA) - Tamoxifen
\end{tabular} & $\begin{array}{l}\text { Inhibiting } \\
\text { P-glycoprotein \& CYP3A4 }\end{array}$ \\
\hline Rivaroxaban & \begin{tabular}{|} 
CHT \\
• Doxorubicin \\
• Vinblastine \\
HT (AA) - Enzalutamide \\
ST - Dexamethasone
\end{tabular} & $\begin{array}{l}\text { Inducing } \\
\text { P-glycoprotein \& CYP3A4 }\end{array}$ & \begin{tabular}{|c|} 
TT (TKI) \\
$\bullet$ Imatinib \\
$\bullet$ Dasatinib \\
$\bullet$ Lapatinib \\
• Nilotinib \\
• Sunitinib \\
HT (ERA) - Tamoxifen
\end{tabular} & $\begin{array}{l}\text { Inhibiting } \\
\text { P-glycoprotein \& CYP3A4 }\end{array}$ \\
\hline Dabigatran & \begin{tabular}{|c|} 
CHT \\
• Doxorubicin \\
・ Vinblastine \\
HT (AA) - Enzalutamide \\
ST - Dexamethasone
\end{tabular} & $\begin{array}{l}\text { Inducing } \\
\text { P-glycoprotein }\end{array}$ & \begin{tabular}{|l|} 
TT (TKI) \\
$\quad$ Lapatinib \\
$\bullet$ Nilotinib \\
• Sunitinib \\
HT (ERA) - Tamoxifen
\end{tabular} & $\begin{array}{l}\text { Inhibiting } \\
\text { P-glycoprotein }\end{array}$ \\
\hline
\end{tabular}

In the evolution of treatment options for VTE, the emergence of novel oral anticoagulants (NOACs), such as rivaroxaban, edoxaban, and apixaban (direct factor Xa inhibitors) has provided important advantages ${ }^{[13]}$. 
These benefits include convenient, oral route of delivery, simple dosing schedule, and no requirement of frequent laboratory parameters monitoring ${ }^{[14]}$. In this way, NOACs are more acceptable to many patients and positively influence their adherence to treatment. Also, NOACs have comparable efficacy and safety profiles to LMWH.

However, NOACs may be inappropriate for some oncology patients, who have a high risk of major bleeding, renal failure, abnormal body mass (overweight or underweight), and GI tumors ${ }^{[14]}$. Potential pharmacologic interactions between some commonly used antineoplastic therapies and NOACs are summarized in Table $1^{[15]}$. Some useful tips to help solve difficulties that are frequently encountered in the care of patients with cancer-associated thrombosis (e.g., recurrent VTE and bleeding) are outlined in Table $2^{[7,16,17]}$. In addition, Figure 1 presents important considerations for making individualized anticoagulation choices, based on outcomes of the pertinent RCTs (CATCH, CLOT, Hokusai VTE Cancer, SELECT-D, ADAM VTE, Caravaggio, AVERT, and CASSINI ${ }^{[18-25]}$.

Table 2. Useful tips for solving difficulties in the care of patients with CAT

\begin{tabular}{|c|c|c|}
\hline \multicolumn{3}{|c|}{ Useful tips for solving difficulties in the care of patients with CAT $^{[7,16,17]}$} \\
\hline $\begin{array}{l}\text { When anticoagulation is needed in } \\
\text { patients with high VTE risk }\end{array}$ & $\begin{array}{c}\text { When anticoagulation is complicated } \\
\text { by bleeding }\end{array}$ & \begin{tabular}{|c|} 
When anticoagulation is \\
complicated by thrombocytopenia
\end{tabular} \\
\hline $\begin{array}{l}\text { Physicians need to evaluate VTE risk in } \\
\text { individual Pt's \& make decisions about } \\
\text { the use of anticoagulation, considering } \\
\text { - previous VTE episodes, } \\
\text { • ca origin/progression, } \\
\text { - medical comorbidities, } \\
\text { • performance status, } \\
\text { - possible bleeding complications }\end{array}$ & $\begin{array}{c}\text { Assess each bleeding episode individually } \\
\text { to identify } \\
\cdot \text { bleeding source, } \\
\bullet \text { severity, } \\
\cdot \text { systemic impact, } \\
\cdot \text { potential reversibility }\end{array}$ & $\begin{array}{c}\text { For platelet count }>50 \times 10^{9} \mathrm{~L}^{-1}-\text { give full } \\
\text { therapeutic dose of LMWH } \\
\text { In the case of acute CAT \& severe } \\
\text { thrombocytopenia }\left(<50 \times 10^{9} \mathrm{~L}^{-1}\right) \text { (if } \\
\text { higher risk of thrombus progression) - give } \\
\text { full-dose anticoagulation (LMWH/UFH) } \\
\text { with platelet transfusion support to maintain } \\
\text { a platelet count of } \geqslant 40-50 \times 10^{9} \mathrm{~L}^{-1}\end{array}$ \\
\hline $\begin{array}{l}\text { Pts with active ca, who are hospitalized } \\
\text { (e.g., due to an acute illness, for } \\
\text { major surgery, or systemic CHT) } \\
\text { should be offered anticoagulation } \\
\text { (except from active bleeding or other } \\
\text { contraindications); medical teams need to } \\
\text { monitor possible AEs of anticoagulation }\end{array}$ & $\begin{array}{l}\text { Provide supportive measures to stop } \\
\text { bleeding, e.g., blood transfusion if } \\
\text { necessary }\end{array}$ & $\begin{array}{c}\text { In the case of acute CAT \& platelet count } \\
<50 \times 10^{9} \mathrm{~L}^{-1} \text { (if lower risk of thrombus } \\
\text { progression) } \\
\text { - reduce the dose of LMWH to } 50 \% \text { of } \\
\text { the therapeutic dose or use a prophylactic } \\
\text { dose of LMWH in pts with a platelet } \\
\text { count of } 25-50 \times 10^{9} \mathrm{~L}^{-1} \\
\text { - temporarily discontinue anticoagulation } \\
\text { in pts while the platelet count is }<25 \times \\
10^{9} \mathrm{~L}^{-1} \\
\text { resume full-dose } \mathrm{LMWH} \text { when } \\
\text { the platelet count is }>50 \times 10^{9} \mathrm{~L}^{-1} \\
\text { without transfusion support, if no } \\
\text { contraindications }\end{array}$ \\
\hline $\begin{array}{l}\text { Prior to initiating systemic CHT, } \\
\text { anticoagulation with NOACs (e.g., } \\
\text { apixaban, rivaroxaban) or LMWH should } \\
\text { be considered, if there is no risk of } \\
\text { bleeding or drug interactions. Pts with ca } \\
\text { undergoing major surgery should receive } \\
\text { pharmacologic thromboprophylaxis } \\
\text { with UFH or LMWH (unless there are } \\
\text { bleeding contraindications); } \\
\text { In some Pts (e.g., with restricted mobility } \\
\text { or obesity) LMWH should be used for } \\
\text { up to } 1 \text { m after open or laparoscopic } \\
\text { abdominal/pelvic surgery }\end{array}$ & $\begin{array}{c}\text { For a major or life-threatening bleeding } \\
\text { episode } \\
\text { • withhold anticoagulation } \\
\text { - consider IVC filter insertion in patients } \\
\text { with acute or subacute CAT (e.g., in pts } \\
\text { with contraindications to anticoagulation } \\
\& \text { a high risk for fatal PE } \\
\text { - do not insert IVC filter in those with } \\
\text { chronic CAT } \\
\text { After bleeding has resolved, remove } \\
\text { retrievable IVC filter \& restart } \\
\text { anticoagulation with LMWH }\end{array}$ & $\begin{array}{l}\text { In the case of subacute or chronic CAT } \\
(>30 \text { days since the index VTE) \& } \\
\text { thrombocytopenia }\left(<50 \times 10^{9} \mathrm{~L}^{-1}\right) \\
\text { - reduce the dose of LMWH to } 50 \% \text { of } \\
\text { the therapeutic dose or use a prophylactic } \\
\text { dose of LMWH in pts with a platelet } \\
\text { count of } 25-50 \times 10^{9} \mathrm{~L}^{-1} \\
\text { - temporarily discontinue anticoagulation } \\
\text { in pts while the platelet count is }<25 \times \\
10^{9} \mathrm{~L}^{-1} \\
\text { - resume full-dose LMWH, as indicated, } \\
\text { when the platelet count is }>50 \times 10^{9} \mathrm{~L}^{-1} \\
\text { without transfusion support }\end{array}$ \\
\hline $\begin{array}{l}\text { pulmonary embolism; pts, patients; UF } \\
\text { symptomatic proximal lower limb DVT } \\
\text { proximal pulmonary artery); Acute CAT }\end{array}$ & c th & $\begin{array}{l}\text { herapy; dgn, diagnosis; IVC, inferior vena } \\
\text {-molecular-weight heparin; m, month; PE, } \\
\text { romboembolism; the definition of CAT = } \\
\text { sels) or PE (including a segmental or more } \\
\text { within the past } 1 \mathrm{~m} \text {; Subacute CAT }=\text { dgn } \\
\text { ex DVT or PE was made }>3 \text { ms ago }\end{array}$ \\
\hline
\end{tabular}




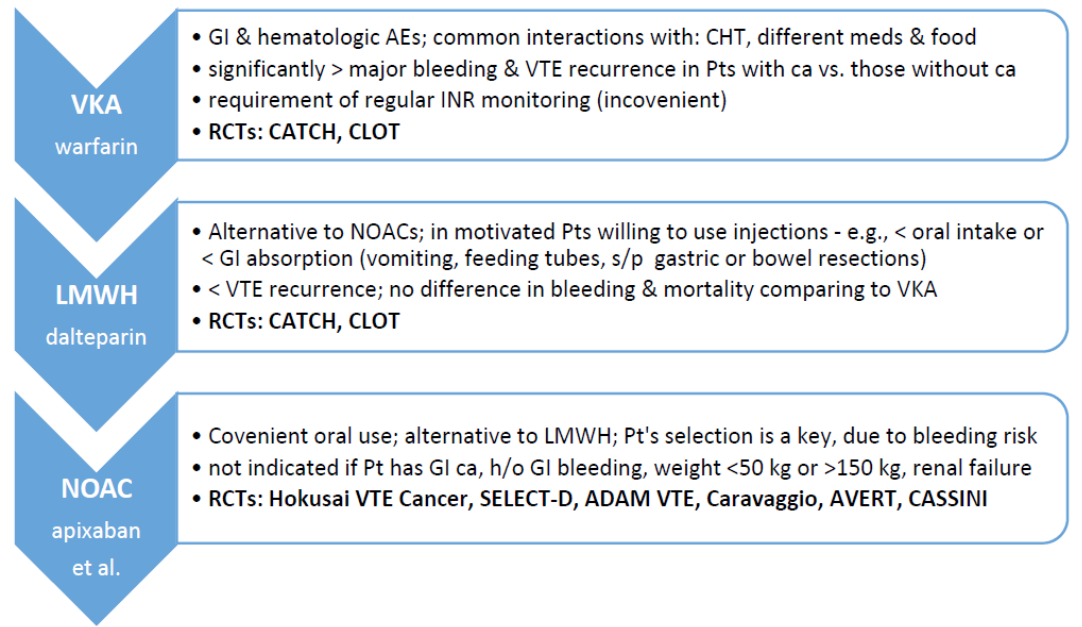

Figure 1. Important considerations for anticoagulation therapy in patients with cancer-related venous thromboembolism - helpful hints for individualized clinical decision-making ${ }^{[18-25]}$.

\section{WHAT FACTORS SHOULD BE INCLUDED, WHEN ADDRESSING ANTICOAGULATION IN THE FINAL STAGE OF LIFE, AMONG PATIENTS WITH CANCER-RELATED VTE?}

VTE guidelines indicate anticoagulation for patients with active cancer, without a specific focus on the anticoagulation management, at the end-of-life scenario. In fact, many patients in their terminal phase of cancer present an elevated thrombotic potential, because of malignancy progression, immobilization, dehydration, inflammation, and various infections ${ }^{[26]}$. Therefore, in such patients with advanced cancers, the anticoagulation should be terminated if their prognosis is very short (e.g. a few days), in order to reduce potential bleeding, without increasing a recurrence of VTE. Also, if such patients develop VTE in the terminal stage of malignancy, comfort care measures can be applied for symptomatic control, without augmenting the bleeding risk. Unquestionably, it is critically important to prevent VTE among inpatients in acute care hospitals. However, in patients using PC services, symptomatic DVT, and PE usually manifest themselves approximately one week, and three weeks, respectively, after the initial pro-thrombotic triggering event ${ }^{[26]}$.

Of course, there can be some patients at the PC setting, who could benefit from different forms of VTE therapy, and thus, specific factors, leading to temporary increases in thrombotic risk and bleeding risk, as well as the patient's point of view should be taken into account. Also, a real need for anticoagulation should be reviewed on a daily basis. VTE prophylaxis should not be offered to patients in the last days of life ${ }^{[27]}$.

\section{WARNING OF THE BLEEDING RISK AMONG TERMINALLY ILL ONCOLOGY PATIENTS HOSPITALIZED IN PC SETTING}

An elevated risk of bleeding represents a great danger in the population of terminally ill patients with cancer. According to data from the RIETE registry (including near three thousand patients with cancer), the renal insufficiency, metastatic progress, recent systemic bleeding, and immobilization (for over four days) were found to be related to a higher risk for the fatal PE and fatal bleeding ${ }^{[28]}$. Furthermore, a low body mass (below 60 $\mathrm{kg}$ ) was another contributor of lethal hemorrhage. In reality, many patients admitted to PC services suffer from a constellation of these conditions, and thus, the treatment teams should be particularly vigilant to the elevated bleeding risk, when making decisions about the VTE prevention in this group. According to a small, prospective randomized study, examining prophylactic anticoagulation (LMWH (nadroparin) vs. placebo) in patients with cancer in the PC setting (with an estimated life expectancy below six months), one VTE and one major bleeding event was reported in the group receiving LMWH, while two minor bleedings episodes were noted in the placebo 
group ${ }^{[29]}$. Based on this study, a decision to apply prophylactic LMWH for individual oncology patients in the PC setting should be carefully considered ${ }^{[29]}$. However, a potential impact of prophylactic LMWH on the patient's survival merits further investigations.

A recent large prospective observational RHESO study has examined the bleeding risk of terminally ill patients, admitted to PC units in France, where 44\% of them were given VTE prophylaxis with LMWH or fondaparinux ${ }^{[30]}$. The rate of major bleeding and clinically relevant non-major bleeding, was $9.8 \%$ (during three months) ${ }^{[30]}$. Bleeding was reported in $11 \%$ of patients who received anticoagulation vs. $8.4 \%$ of those, who did not. Overall, advanced cancer, recent bleeding episode, antiplatelet therapy, and VTE prophylaxis were shown to be independent risk factors for serious bleeding ${ }^{[30]}$. The RHESO study data may indicate that the bleeding risk of VTE prophylaxis can outweigh the benefits in this population ${ }^{[30]}$.

\section{CHALLENGES TO CURRENT RECOMMENDATIONS FOR THE PREVENTION OF VTE IN PATIENTS WITH ADVANCED CANCERS}

Since the application of VTE prophylaxis depends, to a large degree, on the selection of the place of admission (e.g., acute vs. PC services), it still remains undetermined whether or not, the hospital treatment by using VTE prophylaxis will translate to over-aggressive therapy, causing unnecessary exposure of hospitalized, terminally ill oncology patients to the adverse effects of anticoagulation. On the other hand, it is still unclear if some patients admitted to the PC setting may suffer from under-treatment, due to not receiving anticoagulation. At this point, the results of the HIDDen and the RHESO study may question the utility of VTE prophylaxis, among a group of oncology patients with a short prognosis and poor performance status, who can be appropriate candidates for admissions to PC setting ${ }^{[9,30]}$.

\section{ADDRESSING FUTURE DIRECTIONS TO REFINE THE APPROACHES TO LATE-STAGE CAT}

The oncology patients, who require PC services represent a heterogeneous group that can be subdivided into various categories, depending on different types of cancers (e.g., pancreatic, lung, or brain), stages (e.g., metastatic), or medical contexts (e.g., comorbidities) ${ }^{[31]}$. Furthermore, the patient's clinical scenarios and mechanisms of CAT can change in a very dynamic manner ${ }^{[31]}$. For instance, some of the patients, who might have been treated with the curative intent, subsequently enter the PC setting, when their improved survival is no longer a viable option (e.g., after they had succumbed to a terminal stage of cancer).

Of course, such a deterioration requires a flexible approach to address some modifiable factors (e.g., elevated levels of leukocytes or platelets), which can aggravate CAT.

For instance, neutrophils may increase VTE in some oncology patients, via releasing neutrophil extracellular traps (NETs), while monocytes can express tissue factor (TF) ${ }^{[31]}$. As a consequence, pancreatic tumor cells often release TF-positive microvesicles (MVs) into the circulation, which can trigger thrombosis in patients with pancreatic cancer ${ }^{[31]}$. Similarly, tumor-derived granulocyte colony-stimulating factor (G-CSF) (leading to elevation of neutrophils) can cause NETs release and aggravation of thrombosis in patients with lung cancer $^{[31]}$.

In addition, P-selectin expression by the endothelium can deteriorate VTE, via recruiting leukocytes, especially in patients with pancreatic or brain cancer ${ }^{[31]}$. Moreover, brain tumor cells can release podoplanin (PDPN), and PDPN-positive MVs may stimulate circulating platelets, contributing to increased risk of VTE in patients with brain cancer ${ }^{[32]}$. Perhaps, addressing some of the above-mentioned factors of CAT may help refine the individualized oncology patient care in the future. It can be expected that deeper insights into the various 
pathways and triggers of CAT among oncology patients could shed some light on the development of novel strategies that will improve outcomes in late-stage CAT.

\section{THE ROLE OF PATIENT'S PREFERENCES IN THE SHARED DECISION-MAKING PROCESS}

Since the ASCO guidelines may not address some nuances of anticoagulation in oncology patients who require PC services, the decisions about the application of anticoagulation should be made on an individual basis, considering previous episodes of VTE, origin and progression of cancer, medical comorbidities, and possible bleeding complications ${ }^{[7]}$. To facilitate a disposition process with regard to the level of care for patients with advanced cancers, the patient's preferences need to be included as a key element in the shared decision-making. This should fulfill the patient's needs, especially if the available medical evidence is limited. In addition, educating patients, their families or caregivers about these issues, ahead of time, whenever feasible (and documenting the discussion about risks and benefits of the proposed plan in the medical records), need to be included in the medical management. This approach should facilitate mature, personalized decision-making process, with regard to anticoagulation, especially during the end-of-life stage, among oncology patients ${ }^{[7]}$.

\section{CONCLUSION}

Considering a diversity of oncology patient population, and a variety of anticancer and anticoagulation therapies, it has been obvious that "a one size fits all" strategy does not exist. Therefore, the managing of CAT should be conducted according to the current recommendations, considering both modern medical parameters, and personal preferences of patients admitted to the hospital or PC setting for further treatment. In line with this approach, especially in the acute care setting, using NOACs, in the cases of various cancers, when lowering the rate of VTE recurrence is of utmost importance (but keeping in mind the bleeding risk) appears reasonable.

Simultaneously, applying LMWH in the case of gastrointestinal and urothelial cancers (rather than NOACs, due to possible bleeding adverse effects) should be preferred. Due to the scarcity of exact guidelines on anticoagulation, tailored to the group of oncology patients, who require end-of-life care, a "flexible" approach of medical providers is essential. For instance, providers should be able to empathize with the patient's personalized choices, particularly at the transition from the acute to the supportive type of care, when the QoL becomes superior to prolonging the lifespan. Recently, it has been suggested that VTE can be attributed to an advanced neoplastic disease, rather than be a cause of premature death or source of symptom burden deteriorating the QoL. Under these circumstances, thromboprophylaxis for patients with advanced cancers and poor performance status does not appear to be beneficial. Therefore, considering termination of anticoagulation, upon the agreement between the patient's view and the treating clinician's judgment seems justified.

In summary, precise guidelines on VTE prevention and treatment for patients with advanced cancers and poor performance status, who are approaching the final stage of life are certainly needed. Such guidelines should be created, according to the findings of large, prospective RCTs (considering the malignancy type and stage, goals of therapy, performance status, and predicted duration of lifespan) conducted on this patient population. Simultaneously, psychophysical and spiritual needs of the patient and family or caregivers should be expressed clearly and integrated into the final decision-making process.

Abbreviations: AEs, adverse events; CA, cancer; CHT, chemotherapy; < , decreased; GI, gastrointestinal; h/ o; history of; >, increased; INR, international normalized ratio; LMWH, low molecular weight heparin; NOAC, novel oral anticoagulant (apixaban, rivaroxaban, edoxaban); Pt, Patient; RCTs, Randomized Clinical Trials; s/p, status post; VKA, vitamin K antagonist; VTE, venous thromboembolism. 


\section{REFERENCES}

[1] Shojania KG, Duncan BW, McDonald KM, Wachter RM, Markowitz AJ Making health care safer: a critical analysis of patient safety practices. Evid Rep Technol Assess (Summ). 2001; (1-668.): i-x

[2] Khorana AA, Carrier M, Garcia DA, Lee AY. Guidance for the prevention and treatment of cancer-associated venous thromboembolism. J Thromb Thrombolysis. 2016; 41: 81-91.

[3] Carrier M, Khorana AA, Moretto P, Le Gal G, Karp R, Zwicker JI. Lack of evidence to support thromboprophylaxis in hospitalized medical patients with cancer. Am J Med. 2014; 127: 82-86.e1.

[4] Rocque GB, Barnett AE, Illig LC, et al. Inpatient hospitalization of oncology patients: are we missing an opportunity for end-of-life care? J Oncol Pract. 2013; 9: 51-54.

[5] Noble S, Johnson M. Finding the evidence for thromboprophylaxis in palliative care: first let us agree on the question. Palliat Med. 2010; 24: 359-361.

[6] Johnson MJ, McMillan B, Fairhurst C, et al. Primary thromboprophylaxis in hospices: the association between risk of venous thromboembolism and development of symptoms. J Pain Symptom Manage. 2014; 48: 56-64.

[7] Key NS, Khorana AA, Kuderer NM, et al. Venous thromboembolism prophylaxis and treatment in patients with cancer: ASCO clinical practice guideline update. J Clin Oncol. 2019:JCO1901461. https://ascopubs.org/ doi/full/10.1200/JCO.19.01461. Accessed December 30, 2020.

[8] Noble S. Venous thromboembolism and palliative care. Clin Med (Lond). 2019;19(4):315-318. doi:10.7861/ clinmedicine.19-4-315.

[9] White C, Noble S, Watson M, et al. Prevalence, symptom burden, and natural history of deep vein thrombosis in people with advanced cancer in specialist palliative care units (HIDDen): a prospective longitudinal observational study. Lancet Haematol. 2019;6:e79-88.

[10] Lyons P, Verne J. Pattern of hospital admission in the final year of life. BMJ Support Palliat Care. 2011; $1: 81$ 82.

[11] Macbeth F, Noble S, Evans J. Randomized phase III trial of standard therapy plus low molecular weight heparin in patients with lung cancer: FRAGMATIC Trial. J Clin Oncol. 2016;34:488-494.

[12] Maraveyas A, Waters J, Roy R. Gemcitabine versus gemcitabine plus dalteparin thromboprophylaxis in pancreatic cancer. Eur J Cancer. 2012;48:1283-1292.

[13] Al-Samkari H, Connors JM. The role of direct oral anticoagulants in treatment of cancer-associated thrombosis. Cancers (Basel). 2018;10(8).

[14] Ay C , Beyer-Westendorf J, Pabinger I. Treatment of cancer-associated venous thromboembolism in the age of direct oral anticoagulants. Ann Oncol. 2019; 30(6): 897-907.

[15] Lee AY, Carrier M. Treatment of cancer-associated thrombosis: perspectives on the use of novel oral anticoagulants. Thromb Res. 2014;133 Suppl 2:S167-71.

[16] Samuelson Bannow BT, Lee A, Khorana AA, Zwicker JI, Noble S, Ay C, Carrier M. Management of cancerassociated thrombosis in patients with thrombocytopenia: guidance from the SSC of the ISTH. J Thromb Haemost. 2018;16(6):1246-1249.

[17] Carrier M, Khorana AA, Zwicker J, et al. Management of challenging cases of patients with cancer-associated thrombosis including recurrent thrombosis and bleeding: guidance from the SSC of the ISTH. J Thromb Haemost. 2013;11:1760-5.

[18] Lee AY, Kamphuisen PW, Meyer G, et al. Tinzaparin vs Warfarin for treatment of acute venous thromboembolism in patients with active cancer: a randomized clinical trial. JAMA. 2015;314(7):677-686.

[19] Lee AY, Levine MN, Baker RI, et al. Low-molecular-weight heparin versus a coumarin for the prevention of recurrent venous thromboembolism in patients with cancer. N Engl J Med. 2003;349(2):146-153.

[20] Raskob GE, van Es N, Verhamme P, et al; Hokusai VTE Cancer Investigators. Edoxaban for the treatment of cancer-associated venous thromboembolism. N Engl J Med. 2018;378:615-624.

[21] Young AM, Marshall A, Thirlwall J, et al. Comparison of an oral factor Xa inhibitor with low molecular 
weight heparin in patients with cancer with venous thromboembolism: results of a randomized trial (SELECT-D). J Clin Oncol. 2018:36:2017-2023.

[22] McBane RD 2nd, Wysokinski WE, Le-Rademacher JG. Apixaban and dalteparin in active malignancyassociated venous thromboembolism: the ADAM VTE trial. J Thromb Haemost. 2020;18:411-421.

[23] Agnelli G, Becattini C, Meyer G, et al; Caravaggio Investigators. Apixaban for the treatment of venous thromboembolism associated with cancer. N Engl J Med. 2020;382(17):1599-1607.

[24] Kimpton M, Wells PS, Carrier M. Apixaban for the prevention of venous thromboembolism in high-risk ambulatory cancer patients receiving chemotherapy: Rational and design of the AVERT trial. Thromb Res. 2018;164:S124-S129.

[25] Khorana AA, Vadhan-Raj S, Kuderer NM, et al. Rivaroxaban for preventing venous thromboembolism in high-risk ambulatory patients with cancer: rationale and design of the CASSINI trial. Rationale and design of the CASSINI trial. Thromb Haemost. 2017;117:2135-2145.

[26] Noble S, Banerjee S, Pease N. Anticoagulation for cancer associated thrombosis at the end of life: review of a case series of 214 patients. Palliat Med. 2018;32(1S):47-8.

[27] Venous Thromboembolism in over 16s: Reducing the Risk of Hospital-Acquired Deep Vein Thrombosis or Pulmonary Embolism. National Institute for Health and Care Excellence (NICE); London, UK: 2018. [(accessed on 30 December 2020)]. Available online: https://www.nice.org.uk/guidance/ng89.

[28] Monreal M., Falga C., Valdes M., Suarez C., Gabriel F., Tolosa C., Montes J. Fatal pulmonary embolism and fatal bleeding in cancer patients with venous thromboembolism: Findings from the RIETE registry. J. Thromb. Haemost. 2006;4:1950-1956.

[29] Weber C., Merminod T., Herrmann F.R., Zulian G.B. Prophylactic anti-coagulation in cancer palliative care: A prospective randomised study. Support. Care Cancer. 2008;16:847-852.

[30] Tardy B, Piccard S, Guirimand F, et al. Bleeding risk of terminally ill patients hospitalized in palliative care units: the RHESO study. J Thromb Haemost. 2017; 15: 420-428.

[31] Hisada Y, Mackman N. Cancer-associated pathways and biomarkers of venous thrombosis. Blood. 2017;130(13):1499-1506.

[32] Riedl J, Preusser M, Nazari PM, et al. Podoplanin expression in primary brain tumors induces platelet aggregation and increases risk of venous thromboembolism. Blood. 2017;129(13):1831-1839. 